\title{
Analysis of Goniophotometric Reflection Curves
}

\author{
Isadore Nimeroff
}

\begin{abstract}
Surface appearance, apart from texture, is defined by the goniophotometric curve giving the angular distribution of the reflected light, but a summary of current goniophotometric techniques shows that essential parts of these fundamental data have been neglected. An established method of statistics is applied to the problem of goniophotometric-curve analysis and interpretation whereby such data are reduced to dispersion, skewness, and kurtosis indexes. The kurtosis index is shown to correlate better with subjective estimates of the distinctness of the reflected images than the conventional measure, $60^{\circ}$ specular gloss. The kurtosis index is applied to one problem in the selection of optimum glossmeter geometry. 지
\end{abstract}

\section{Introduction}

In pursuit of a method for specifying glossiness, or luster, of surfaces many theorists, researchers, and engineers, physical and psychophysical, have devoted thought and time to theorizing and experimenting. Techniques for measurement have been devised, ranging from observation with a single set to observations involving many sets of illuminating and viewing conditions. The former of these techniques is an oversimplified solution to the problem; the latter is too costly and time consuming to perform for every surface under consideration. Such a complete set of data was published by Thaler [1] ${ }^{1}$ for a series of magnesium oxide surfaces. A compromise between these extremes is required. Such a compromise involves obtaining goniophotometric curves near the angle of specular reflection in the plane of measurement for one unidirectional incidence angle. ${ }^{2}$

\section{Goniophotometers}

The earliest known goniophotometer is the one devised by Bouguer [2] in attempting to corroborate Lambert's law [3] by experimental evidence. Since then many goniophotometers have been developed and described. The problem of how to analyze and interpret the curves obtained on goniophotometers still remains. This paper proposes using an established statistical method to analyze monoplane goniophotometric reflection curves, yielding numerics that correlate with specular gloss and estimates of distinctness-of-image gloss.

Many workers in the field of glossimetry have realized that the only way to specify completely the optical properties of reflecting surfaces is by analyzing goniophotometric curves of the reflected light. In the interest of economy one goniophotometric reflection curve for one angle of incidence and one plane of view may provide an adequate account of what happens to the flux reflected by a surface. This flux distribution can be measured visually or photoelectrically. The most detailed description of a visual goniophotometer is given by McNicholas [4]. His instrument had provision for diffuse and unidi-

1 Figures in brackets indicate the literature references at the end of this paper. 2 Photometric definitions and terms used here are contained in a paper by Hammond and Nimeroff [6]. rectional illumination and multiplane view. $\mathrm{He}$ used his goniophotometer to obtain distribution curves for diffusing media.

Wetlaufer and Scott [5] described a photoelectric goniophotometer with rectangular source and receiver apertures summing to $50^{\prime}$ by $5.5^{\circ}$, but the relative sizes of these apertures were not given. They obtained goniophotometric data near the angle of specular reflection with $45^{\circ}$ incidence for 13 materials exhibiting reflection ranging from nearly completely specular to nearly completely diffuse, and drew conclusions about the relative merits of large and small incidence angles in the measurement of specular gloss. Only one incidence angle was used, and no analysis of the goniophotometric data was made.

In a paper on $60^{\circ}$ specular-gloss measurement [6] Hammond and Nimeroff described the optics of a photoelectronic monoplane goniophotometer having high resolution, $0.3^{\circ}$ circular source, and $0.6^{\circ}$ circular receiver apertures. With this instrument fractional reflectance curves were obtained for a series of specimens obviously differing in appearance, yet having approximately the same $60^{\circ}$ specular-gloss readings according to the ASTM Method [7]. ${ }^{3}$ The curves indicated that these specimens had radically different fractional-reflectance distributions. If the information contained in these curves could be adequately expressed as a single number, a better index of gloss characteristics would be obtained than is possible from the simple measurement of specular gloss.

\section{The Concept of Moments}

A method for the description of these fractionalreflectance curves may be found in the field of statistics, where the technique of characterizing various types of frequency distributions is of fundamental importance. In statistics the measures of dispersion, skewness, and peakedness have been found to facilitate comparisons between distributions. These measures are computed by the use of moments. A brief résumé of the concept of moments follows.

With observed frequencies, $f_{1}, f_{2}, \ldots . ., f_{n}$, corresponding to variables, $x_{1}, x_{2}, \ldots . . x_{n}$, and with the sum of the frequencies, $f_{1}+f_{2}+. .+f_{n}$ equal

\footnotetext{
${ }^{3}$ Wherever $60^{\circ}$ specular gloss is mentioned this method is implied.
} 
to $\sum_{1}^{n} f_{i}$, we define the $k$ th moment,

$$
\mu_{k} \equiv \frac{\sum_{1}^{n} f_{i} x_{i}^{k}}{\sum_{1}^{n} f_{i}}=\frac{\sum_{1}^{n} f_{i} x_{i}^{k}}{N},
$$

where $N=\sum_{1}^{n} i f_{i} . \quad$ In other words, the $k$ th moment is the arithmetic mean of the $k$ th powers of the variate, $x_{i}$. When the axis of moments is taken about the centroid of mean, $\bar{x}$, or the variable $x_{i}$ then,

$$
\pi_{k}=\frac{\sum_{1}^{n} f_{i}\left(x_{i}-\bar{x}\right)^{k}}{N}
$$

is the $k$ th monent about the centroid, or mean.

\section{Curve Analysis}

Harrison [8] suggests that polar-intensity distribution curves should be analyzed to obtain the desired information about surface appearance. He suggests also that these polar curves should not be considered as composed of ellipses, circles, and straight lines, because the specular components (lines) cannot be separated physically from the diffuse components (circles). Barkas [9] suggests a method for theoretically separating these two components for fairly matte surfaces by substituting an equivalent surface for a physical surface. The Barkas treatment has not yet been applied to semiglossy and glossy surfaces.

Pokrowski [10] found that by summing the diffuse reflection and the specular-reflection components one could obtain for a given angle of illumination the distribution of reflected flux for all directions. The computation of these components is made on the assumption that diffuse reflection obeys Lambert's law and that the specular reflection obeys Fresnel's law [11] for an equal distribution of mirror facets oriented at all possible angles. Chinmayanandam [12] derived an exponential expression to account theoretically for the reflection from optically rough surfaces by assuming that the angles between the reflecting elements and the gross plane are randomly distributed according to the probability law.

Typical goniophotometric curves observed for materials ranging from matte to high gloss are shown in figure 1. Clues as to how to analyze these data are difficult to obtain from a polar graph. However, if rectangular coordinates are used for the data in figure 1 and fractional reflectance, $F$, is plotted on a logarithmic scale as the ordinate against angular departures from the specular angle, $d$, on a linear scale as abscissa (see fig. 2) their resemblance to normal frequency distributions about the arithmetic mean becomes apparent.

In the analysis of frequency distributions, mathematical statistics (see, for example, Reitz [13] and Croxton and Cowden [14]) has shown that three indexes are important. These three indexes are

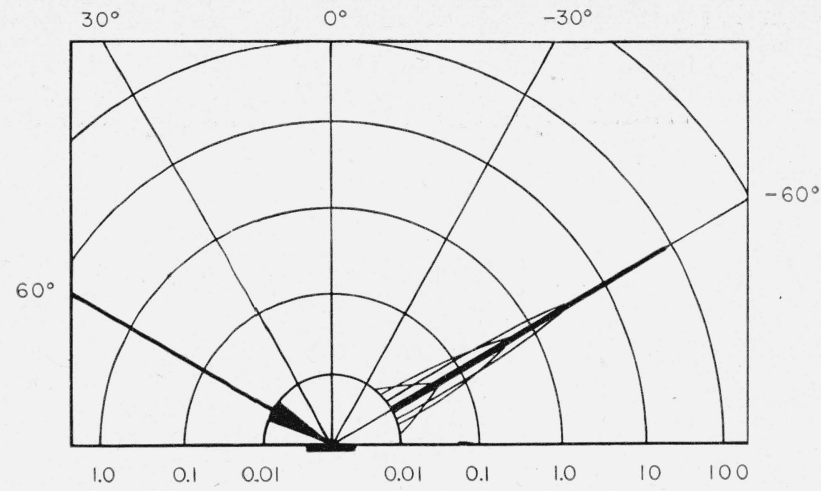

Figure 1. Typical goniophotometric curves on log-polar coordinates.

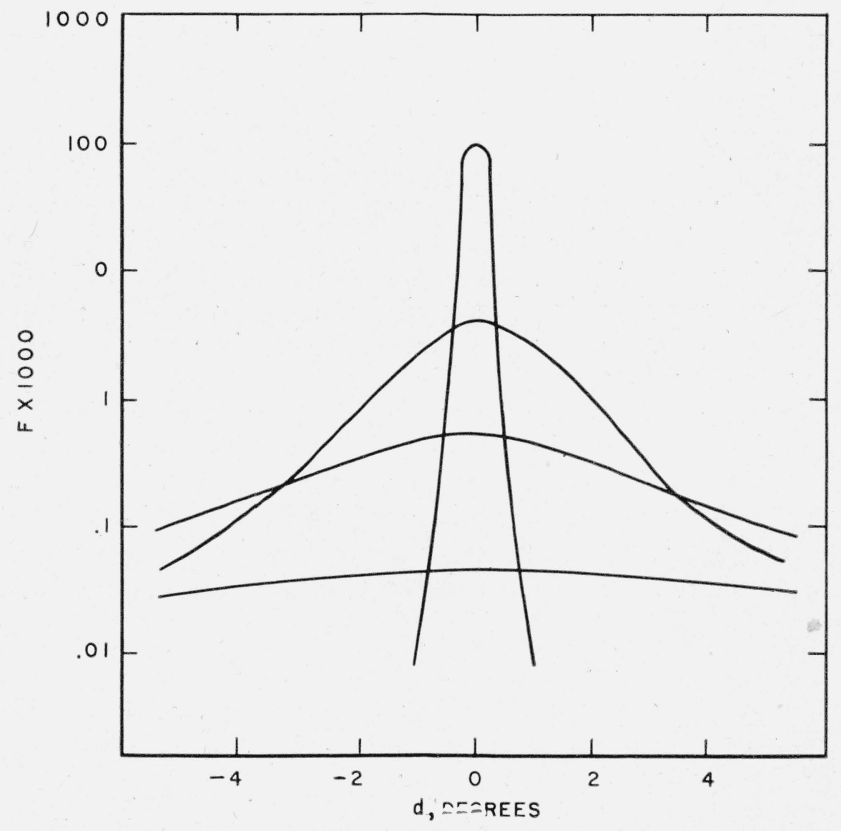

Fiaure 2. Typical goniophotometric curves on log-linear coordinates.

Same data as figure 1.

dispersion index, $\sigma$; skewness index, $\alpha_{3}$; and kurtosis index, $\alpha_{4}$. For the purposes of this paper, they are defined as follows:

$$
\begin{gathered}
\sigma \equiv\left(\pi_{2}\right)^{\frac{1}{2}} \\
\alpha_{2} \equiv \frac{\pi_{3}}{\left(\pi_{2}\right)^{\frac{3}{2}}}=\frac{\pi_{3}}{\sigma^{3}} \\
\alpha_{4} \equiv \frac{\pi_{4}}{\left(\pi_{2}\right)^{2}}=\frac{\pi_{4}}{\sigma^{4}}
\end{gathered}
$$

The first index, $\sigma$, gives an indication of the spread of the distribution, the second, $\alpha_{3}$, gives an indication of the dissymmetry of the distribution about the mean, and the third, $\alpha_{4}$, gives an indication of the peakedness of the distribution. This last index is of primary importance in goniophotometric curve analysis for, as will be shown later, its correlation 
with estimates of distinctness-of-reflected-image gloss is almost perfect.

A method was devised by Pelton [15] in which he obtains a series of measurements of photometric brightness, $B_{\theta}$, by tilting a specimen about an axis in the photometric plane. Thus he obtained a type of goniophotometric distribution curve with simultaneously varying incidence and viewing angles. This curve suggested to him an analogy to the frequency distribution curve according to the law of probability. He then computed the dispersion index, $\sigma$, (usually called standard deviation) using the relation,

$$
\sigma=\sqrt{\frac{\Sigma_{\theta} B_{\theta} \Theta^{2}}{\Sigma_{\theta} B_{\theta}}}
$$

where $\theta$ is the angle of tilt of the specimen. This equation is analogous to taking the square root of eq (1), for which $k=2$. As the standard deviation has an inverse relation to the sharpness of his curves, Pelton took $1 / \sigma$ as a measure of what he called "peakiness."

\subsection{Computation Short Cuts}

To shorten the computation we define deviates, $d_{i}$, from an assumed mean, $x^{\prime}$. Then moments

$$
\nu_{k}=\frac{\sum_{1}^{n} f_{i}\left(d_{i}\right)^{k}}{N}
$$

about $x^{\prime}$ are related to $\pi_{k}$ about the mean by the general expression obtained from the expansion of the binomial in eq (2),

$$
\pi_{k}=\sum_{0}^{k}{ }_{t}\left(\begin{array}{l}
k \\
t
\end{array}\right)\left(-\nu_{1}\right)^{t}\left(\nu_{k-t}\right),
$$

where

$$
\left(\begin{array}{l}
k \\
t
\end{array}\right)=\frac{k !}{t !(k-t) !}
$$

$t$ being an integer having values, $0 \leq t \leq k$.

The first five moments about the mean $\left(\pi_{0}, \pi_{1}, \pi_{2}\right.$, $\pi_{3}$, and $\pi_{4}$ ) are in table 1 . Dispersion, skewness, and kurtosis indexes may then be defined in terms of these moments.

Table 1. Definition of indexes

\begin{tabular}{|l|l|}
\hline$k$ & \multicolumn{1}{|c|}{$\pi_{k}$} \\
\hline 0 & 1 \\
1 & 0 \\
2 & $\nu_{2}-\nu_{1}^{2}$ \\
3 & $\nu_{2}-3 \nu_{1} \nu_{2}+2 \nu_{1}^{3}$ \\
4 & $\nu_{4}-4 \nu_{1} \nu_{3}+6 \nu_{1}^{2} \nu_{2} \times 3 \nu_{1}^{4}$ \\
\hline
\end{tabular}

To facilitate the computation of the three indexes the data were taken at the angle of specular reflection and at $1^{\circ}$ intervals for $5^{\circ}$ on either side of the specular angle. To show the ease of computation an example is shown in table 2 for a plaque of white ceramic tile illuminated at $60^{\circ}$, specular gloss of about 90 . The computation is further simplified, because we can readily take

$$
f_{i} d_{i}^{k}=d_{i} f_{i} d_{i}^{k-1},
$$

where $d_{i}$ is the departure from the specular angle

\begin{tabular}{|c|c|c|c|c|c|c|}
\hline $\begin{array}{l}\text { Angle } \\
\text { (deg) }\end{array}$ & $d_{i}$ & $f_{i}$ & $f_{i} d_{i}$ & $f_{i} d_{i}^{2}$ & $f_{i} d_{i}^{3}$ & $f_{i} d_{i}^{4}$ \\
\hline $\begin{array}{l}55 \\
56 \\
57 \\
58 \\
59 \\
60\end{array}$ & $\begin{array}{r}-5 \\
-4 \\
-3 \\
-2 \\
-1 \\
0\end{array}$ & $\begin{array}{r}0.009 \\
.014 \\
.033 \\
.15 \\
1.45 \\
7.94\end{array}$ & $\begin{array}{c}-0.045 \\
-.056 \\
-.099 \\
-.30 \\
-1.45 \\
0.00\end{array}$ & $\begin{array}{r}0.225 \\
.224 \\
.297 \\
.60 \\
1.45 \\
0.00\end{array}$ & $\begin{array}{c}-1.125 \\
-0.896 \\
-.891 \\
-1.20 \\
-1.45 \\
0.00\end{array}$ & $\begin{array}{l}5.625 \\
3.584 \\
2.673 \\
2.40 \\
1.45 \\
0.00\end{array}$ \\
\hline $\begin{array}{l}61 \\
62 \\
63 \\
64 \\
65\end{array}$ & $\begin{array}{l}1 \\
2 \\
3 \\
4 \\
5\end{array}$ & $\begin{array}{l}2.04 \\
0.26 \\
.053 \\
.019 \\
.010\end{array}$ & $\begin{array}{l}2.04 \\
0.52 \\
.159 \\
.076 \\
.050\end{array}$ & $\begin{array}{l}2.04 \\
1.04 \\
0.477 \\
.304 \\
.250\end{array}$ & $\begin{array}{l}\text { 2. } 04 \\
2.08 \\
1.431 \\
\text { 1. } 216 \\
1.250\end{array}$ & $\begin{array}{l}\text { 2. } 04 \\
\text { 4. } 16 \\
\text { 4. } 293 \\
\text { 4. } 864 \\
\text { 6. } 250\end{array}$ \\
\hline & $\Sigma$ & 11.978 & +0.895 & 6. 907 & +2.455 & 37. 339 \\
\hline \multicolumn{7}{|c|}{$\nu_{1}=\frac{\Sigma f_{i} d_{i}}{N}=+\frac{0.895}{11.978}=0.0747$} \\
\hline \multicolumn{7}{|c|}{$\nu_{2}=\frac{\Sigma f_{i} d_{i}^{2}}{N}=\frac{6.907}{11.978}=0.577$} \\
\hline \multicolumn{7}{|c|}{$\nu_{3}=\frac{\Sigma f_{i} d_{i}^{3}}{N}=+\frac{2.455}{11.978}=0.205$} \\
\hline \multicolumn{7}{|c|}{$\nu_{i}=\frac{\Sigma f_{i} d_{i}^{4}}{N}=\frac{37.339}{11.978}=3.117$} \\
\hline & & \multicolumn{2}{|c|}{$\pi_{2}=\nu_{2}-\nu_{1}^{2}$} & \multicolumn{3}{|l|}{$=0.571$} \\
\hline & & \multicolumn{2}{|c|}{$\pi_{3}=\nu_{3}-3 \nu_{1} \nu_{2}+2 \nu_{1}^{3}$} & \multicolumn{3}{|l|}{$=0.76$} \\
\hline & & \multicolumn{4}{|c|}{$\pi_{4}=\nu_{4}-4 \nu_{1} \nu_{3}+6 \nu_{1}^{2} \nu_{2}-3 \nu_{1}^{4}=3.072$} & \\
\hline & & \multicolumn{2}{|c|}{$\sigma=\left(\pi_{2}\right)^{1 / 2}$} & \multicolumn{2}{|l|}{$=0.756$} & \\
\hline & & \multicolumn{2}{|c|}{$a_{3}=\pi_{3} /\left(\pi_{2}\right)^{3 / 2}$} & \multicolumn{2}{|l|}{$=0.176$} & \\
\hline & & \multicolumn{2}{|c|}{$a_{4}=\pi_{4 /}\left(\pi_{2}\right)^{2}$} & \multicolumn{2}{|l|}{$=9.42$} & \\
\hline
\end{tabular}
(assumed mean), and $f_{i}$ is the goniophotometrically obtained fractional reflectance (distribution frequency).

TABle 2. Computa:ion of indexes

\subsection{Characteristics of the Indexes}

If a distribution is symmetrical, the skewness index, $\alpha_{3}$, has a value of zero. There is no upper limit to the magnitude of $\alpha_{3}$. Mathematically stated,

$$
0 \leq\left|\alpha_{3}\right| \leq \infty .
$$

Values for $\alpha_{3}$ as great as \pm 2 indicate marked skewness, however.

The kurtosis index, $\alpha_{4}$, has a value of 3 for the normal curve, $y=a e^{-x^{2} / 2 \sigma^{2}}$. For curves more peaked than the normal curve, $\alpha_{4}$ is greater than 3, whereas for curves flatter than the normal curve, $\alpha_{4}$ is less than 3. There is no upper limit for $\alpha_{4}$. For a flat curve, that is for $f_{i}$ constant, the value of $\alpha_{4}$, designated $\alpha_{4}^{\prime}$, is determined from the number of observations on which computations are made. From the definitions of $\alpha_{4}, \pi_{4}$, and $\pi_{2}$ we see that

$$
\alpha_{4}^{\prime}=\frac{\pi_{4}}{\left(\pi_{2}\right)^{2}}=\frac{\sum_{1}^{n}{ }_{i} f_{i} d_{i}^{4} / N}{\left(\sum_{1}^{n}{ }_{i} f_{i} d_{i}^{2} / N\right)^{2}}=n \frac{\sum_{1}^{n}{ }_{i} d_{i}^{4}}{\left(\sum_{1}^{n}{ }_{i} d_{i}^{2}\right)^{2}}
$$


where $n=N / f_{i}=$ number of angle settings. The general case for $1^{\circ}$ intervals is shown as follows: We have

$$
\left.\begin{array}{l}
\sum_{1}^{n} d_{i}^{2}=2 \sum_{1}^{M} X_{i}^{2} \\
\sum_{1}^{n}{ }_{i} d_{i}^{4}=2 \sum_{1}^{M}{ }_{i} X_{i}^{4}
\end{array}\right\}
$$

where $X_{i}$ represents positive numbers ranging from 1 to $M$, since observations are made at positive and negative angles. Then, $n=2 M+1$ since $n$ includes $d_{i}=0^{\circ}$. The sums of the first two even powers of the first $M$ natural numbers may be computed from the following equations:

$$
\sum_{1}^{M} X_{i}^{2}=\left(\frac{2 M+1}{3}\right)\left(\frac{M(M+1)}{2}\right)
$$

and

$$
\left.\sum_{1}^{M}{ }_{i} X_{i}^{4}=\left(\frac{3 M^{2}+3 M-1}{5}\right)\left(\frac{2 M+1}{3}\right)\left(\frac{M(M+1)}{2}\right)\right)
$$

Thus

$$
\alpha_{4}^{\prime}=\frac{2(2 M+1)\left(\frac{3 M^{2}+3 M-1}{5}\right)\left(\frac{2 M+1}{3}\right)\left(\frac{M(M+1)}{2}\right)}{4\left(\frac{2 M+1}{3}\right)\left(\frac{M(M+1)}{2}\right)\left(\frac{2 M+1}{3}\right)\left(\frac{M(M+1)}{2}\right)}=\frac{9}{5}-\frac{3}{5 M(M+1)} .
$$

The upper limiting value, $L_{\alpha_{4}^{\prime}}$, of the kurtosis index for a complete diffusor as $M$ increases without bound is

$$
\underset{M \rightarrow \infty}{L_{\alpha_{4}^{\prime}}}=9 / 5=1.8 .
$$

The lower limit occurs when $M=1$, that is when observations are taken at the specular angle and at $\pm 1^{\circ}$. In this case $\alpha_{4}^{\prime}=1.8-0.3=1.5$. Thus we may state, mathematically:

$$
1.5 \leq \alpha_{4}^{\prime} \leq 1.8 \text {. }
$$

For the number of the intervals used in this analysis, that is five intervals on either side of the specular angle, $n=11, M=5$. Thus for a completely diffusing surface, that is, one having constant $f_{i}$

$$
\alpha_{4}^{\prime}=1.80-\frac{3}{25(6)}=1.78 \text {. }
$$

Should we wish to define a scale of peakedness for which a perfect diffuser is assigned the value zero, then we may subtract $\alpha_{4}^{\prime}$ from $\alpha_{4}$, and call the resulting difference the peakedness index, $P$.

Thus

$$
P \equiv \alpha_{4}-\alpha_{4}^{\prime}
$$

If the ordinates of one distribution are a constant factor of the corresponding ordinates of another distribution, then the indexes $\sigma, \alpha_{3}$, and $\alpha_{4}$ for one distribution will equal the corresponding indexes for the other. To distinguish one curve from the other, one point on each curve needs to be specified. A convenient point is the value of the fractional reflectance at the specular angle.

The dispersion index, $\sigma$, is at its lower limit for completely specular reflection, and is large for completely diffuse reflection. The lower limit is zero, because $d_{i}=0^{\circ}$ at the specular angle. The value for completely diffuse reflection is determined as follows :
For completely diffuse reflection, $f_{i}=$ constant,

$$
\sigma=\left(\frac{\sum_{1}^{n}{ }_{i} d_{i}^{2}}{n}\right)^{\frac{1}{2}} .
$$

But from eq 11

$$
\sigma=\left(\frac{2 \sum_{1}^{M} X_{i}}{n}\right)^{\frac{1}{2}} .
$$

Here also $n=2 M+1$, and from eq 12

$$
\sigma=\left(\frac{M(M+1)}{3}\right)^{\frac{1}{2}} .
$$

For the number of the intervals used in this analysis, $M=5$. Thus when $f_{i}$ is constant, $\sigma=3.16$.

The characteristics for the indexes, $\alpha_{4}$ and $\sigma$, lead to conclusions about their utility in describing surface appearance. Consider the ranges of these two indexes for the number of observations used in this analysis. Mathematically stated, from flat to peaked curves, the ranges are $1.78 \leq \alpha_{4} \leq \infty$ and $3.16 \geq \sigma \geq 0$.

When a reflection curve is flat, $\alpha_{4}$ has a small value in its range, while $\sigma$ has a large value in its range. Conversely, when a curve describes a completely specular reflection, $\alpha_{4}$ is at its upper limit, and $\sigma$ is at its lower limit. Thus $\alpha_{4}$ may indicate glossiness directly, and $\sigma$ may indicate glossiness inversely.

\section{Application to Glossimetry}

The practice of glossimetry is now quite well established and generally yields satisfactory discriminations, but must be recognized as an abridged goniophotometric technique. Just as in the problems of colorimetry, the standardization of abridged methods of measurement is necessary to enable one to obtain on a common basis rapid comparisons during production of a specific material. The gen- 
eral method of analyzing goniophotometric distribution curves described in this paper may be the means of arriving at some instrumental geometry and recommended procedures for making objective evaluations of the surface appearance attribute ascribable to gloss.

Subjective observations on the specular gloss of specimens are usually made without use of anglemeasuring devices. Therefore, as the skewness index is a measure of dissymmetry of a distribution, no attempt is made to correlate this index with the surface-appearance attribute ascribable to gloss.

The first problem confronting this analysis is, do the indexes $\alpha_{4}$ and $\sigma$ correlate with surface appearance, specular gloss, and distinctness-of-image gloss, as well as or better than the existing glossimetry techniques? If the correlations prove satisfactory, the next problem is how to interpret this analysis so that instrument geometry for abridged methods may be selected wisely. Three important geometric variables of a glossmeter are (1) angle of incidence, (2) receiver aperture size, (3) the ratio of the angular size of the source to the receiver aperture.

\subsection{Correlation with Distinctness of Reflected Images}

One of the attributes of surface appearance is the distinctness with which images are reflected. This attribute is influenced by at least two properties of specimens. One of the properties is the smoothness of the surface, the other is the diffuse reflection of the specimen. Lack of smoothness tends to distort the reflected images, and diffuse reflection controls the contrast between highlight and shadow.

Heretofore no satisfactory method has been devised to measure objectively distinctness-of-image gloss. Hunter [16] in 1936 described a photographic technique for recording distinctness of reflected images. However, no quantitative analysis was indicated in his paper.

\section{a. Experimental Conditions}

As there is need for a quantitative index of distinctness of reflected images, the kurtosis index was tested for correlation with distinctness of image estimates. For this experiment estimates were made by six observers, ranging in familiarity with the problems from thoroughly experienced to completely naive.

The 22 specimens used were of the following materials: Polished black glass, sand-blasted black glass, acid-etched black glass, polished white Vitrolite, depolished white Vitrolite, and white ceramic tile. These specimens were placed on a black cloth-covered table. The table was placed near a window so that the specimens were illuminated by north-sky light. The estimates made by the observers were on the distinctness of the images of the crossbars of the window. Observers were permitted to turn, tilt, or raise the specimens during the evaluations. The observers arranged the specimens in the order of estimated decreasing distinctness of the reflected images. The resulting data are shown in table 3 , together with the ranking obtained by the kurtosis index, dispersion index, and $60^{\circ}$ specular-gloss values of the specimens.

\section{b. Rank Correlation}

Correlation indexes were computed on the basis of Spearman's rank correlation coefficient, $\rho$. This correlation coefficient has been found quite useful in correlating ordered or ranked data. The equation for computing $\rho$ is

$$
\rho=1-\frac{6 \Sigma D^{2}}{N\left(N^{2}-1\right)}
$$

where $D$ is the rank difference, and $N$ is the number of items.

This equation indicates by its sign whether the correlation is direct $(+)$ or inverse $(-)$. Whenever there is a tie in rank, the positions are split among the different items in the tie.

TABLE 3. Rank order according to distinctness of image esimates, peakedness, $60^{\circ}$ gloss, and dispersion

\begin{tabular}{|c|c|c|c|c|c|c|c|c|c|}
\hline \multirow{2}{*}{$\begin{array}{l}\text { Speci- } \\
\text { men }^{1}\end{array}$} & \multicolumn{6}{|c|}{ Observers } & \multirow{2}{*}{$\begin{array}{l}\text { De- } \\
\text { creas- } \\
\text { ing } \\
\text { peak- } \\
\text { edness }\end{array}$} & \multirow{2}{*}{$\begin{array}{l}\text { De- } \\
\text { creas- } \\
\text { ing } \\
60^{\circ} \\
\text { gloss }\end{array}$} & \multirow{2}{*}{$\begin{array}{l}\text { In- } \\
\text { creas- } \\
\text { ing } \\
\text { dis- } \\
\text { persion }\end{array}$} \\
\hline & M MB & DBJ & $\mathrm{HKH}$ & $\mathrm{GWH}$ & IN & $\mathrm{HS}$ & & & \\
\hline B94 & 1 & 1 & 1 & 1 & 1 & 1 & 1 & 2 & 1 \\
\hline V90 & 7 & 6 & 6 & 7 & 7 & 7 & 2 & 3 & 2 \\
\hline $\mathrm{S} 1$ & 2 & 2 & 2 & 2 & 2 & 2 & 3 & 5 & 5 \\
\hline $\mathrm{S} 2$ & 3 & 3 & 3 & 3 & 3 & 3 & 4 & 7.5 & 3.5 \\
\hline G102 & 8 & 8 & 8 & 8 & 8 & 8 & 5 & 1 & 3.5 \\
\hline S3 & 4 & 4 & 4 & 4 & 4 & 4 & 6 & 10 & 6 \\
\hline $\mathrm{S} 4$ & 5 & 5 & 5 & 5 & 5 & 5 & 7 & 13 & 7 \\
\hline S5 & 6 & 7 & 7 & 6 & 6 & 6 & 8 & 16.5 & 8 \\
\hline G87 & 10 & 10 & 10 & 11 & 10 & 9 & 9 & 4 & 9 \\
\hline A1 & 9 & 9 & 9 & 9 & 9 & 11 & 10 & 6 & 10 \\
\hline G80 & 13 & 12 & 12 & 13 & 12 & 10 & 11 & 7.5 & 11 \\
\hline A2 & 11 & 11 & 11 & 11 & 10 & 12 & 12 & 9 & 12 \\
\hline G70 & 15 & 14 & 15 & 15 & 15 & 15 & 13 & 11 & 13 \\
\hline A3 & 12 & 13 & 13 & 12 & 13 & 13 & 14 & 12 & 14 \\
\hline $\mathrm{G} 60$ & 17 & 15 & 16 & 16 & 16 & 16 & 15 & 14 & 15 \\
\hline G50 & 16 & 17 & 17 & 17 & 17 & 17 & 16 & 15 & 16 \\
\hline A4 & 14 & 16 & 14 & 14 & 14 & 14 & 17 & 16.5 & 17 \\
\hline $\mathrm{G} 40$ & 18 & 18 & 18 & 18 & 18 & 18 & 18 & 18 & 18 \\
\hline $\mathrm{G} 30$ & 19 & 19 & 19 & 19 & 19 & 19 & 19 & 19 & 19 \\
\hline $\mathrm{G} 20$ & 20 & 20 & 20 & 20 & 20 & 20 & 20 & 20 & 20 \\
\hline $\mathrm{G} 10$ & 21 & 21 & 21 & 21 & 21 & 21 & 21 & 21 & 21 \\
\hline V1 & 22 & 22 & 22 & 22 & 22 & 22 & 22 & 22 & 22 \\
\hline
\end{tabular}

$1 \mathrm{G}$, white ceramic; V, white Vitrolite; B, black glass, polished; A, black glass acid etched; S, black glass, sand-blasted.

The data indicate that confusion exists among the estimates where the specimens have nearly the same distinctness of image, but one is black and the other white. Therefore, computation of $\rho$ was made for the entire group and for the group of black and the group of white specimens.

The correlation indexes obtained by the six observers for the three correlation conditions are shown in table 4 . These results indicate that correlation of image distinctness with $\alpha_{4}$ is better than with $\sigma$ and is far better than with $60^{\circ}$ specular gloss. When the specimens are separated into two groups according to reflectance, then correlation with $\alpha_{4}$ is nearly perfect. We may conclude that the diffuse reflectance of a specimen is an important factor in distinctness-of-image estimates. 
TABLE 4. Summary of correlation coefficients

\begin{tabular}{|c|c|c|c|c|c|c|c|c|c|}
\hline \multirow{3}{*}{ Observer } & \multicolumn{9}{|c|}{ Rank correlation coefficient } \\
\hline & \multicolumn{3}{|c|}{ Black and white } & \multicolumn{3}{|c|}{ Black only } & \multicolumn{3}{|c|}{ White only } \\
\hline & $\alpha_{4}$ & $\sigma$ & $\begin{array}{l}60^{\circ} \\
\text { gloss }\end{array}$ & $\alpha_{4}$ & $\sigma$ & $\begin{array}{c}60^{\circ} \\
\text { gloss }\end{array}$ & $\alpha_{8}$ & $\sigma$ & $\begin{array}{c}60^{\circ} \\
\text { gloss }\end{array}$ \\
\hline $\begin{array}{l}\text { DBJ } \\
\text { HKH } \\
\text { IN } \\
\text { HS } \\
\text { MMB } \\
\text { GWH }\end{array}$ & $\begin{array}{r}0.975 \\
.968 \\
.962 \\
.963 \\
.957 \\
.955\end{array}$ & $\begin{array}{r}0.965 \\
.958 \\
.951 \\
.952 \\
.952 \\
.948\end{array}$ & $\begin{array}{r}0.795 \\
.786 \\
.771 \\
.773 \\
.765 \\
.760\end{array}$ & $\begin{array}{l}1.000 \\
1.000 \\
1.000 \\
1.000 \\
1.000 \\
1.000\end{array}$ & $\begin{array}{r}0.988 \\
.988 \\
.988 \\
.988 \\
.988 \\
.988\end{array}$ & $\begin{array}{r}0.664 \\
.664 \\
.664 \\
.664 \\
.664 \\
.664\end{array}$ & $\begin{array}{l}1.000 \\
1.000 \\
1.000 \\
1.000 \\
1.000 \\
1.000\end{array}$ & $\begin{array}{l}1.000 \\
1.000 \\
1.000 \\
1.000 \\
1.000 \\
1.000\end{array}$ & $\begin{array}{r}0.993 \\
.993 \\
.993 \\
.993 \\
.986 \\
.993\end{array}$ \\
\hline Mean & 0.963 & 0.954 & 0.775 & 1.000 & 0.988 & 0.664 & 1. 000 & 1.000 & 0.992 \\
\hline
\end{tabular}

\subsection{Correlation With $60^{\circ}$ Specular Gloss}

One of the satisfactory glossimetry techniques is the $60^{\circ}$ specular gloss method. If the indexes obtained by analysis of goniophotometric curves correlate with $60^{\circ}$ specular gloss and also yield more information, then this analysis is an improvement over the abridged method. To see if correlation exists between kurtosis and $60^{\circ}$ specular gloss, we plot $\alpha_{4}$ on a logarithmic scale against $60^{\circ}$ speculargloss readings for the 22 specimens listed in table 3 and determine if a smooth curve will fit the resulting points. Figure 3 is a plot of the data showing division into two distinct groups, shown by solid lines,

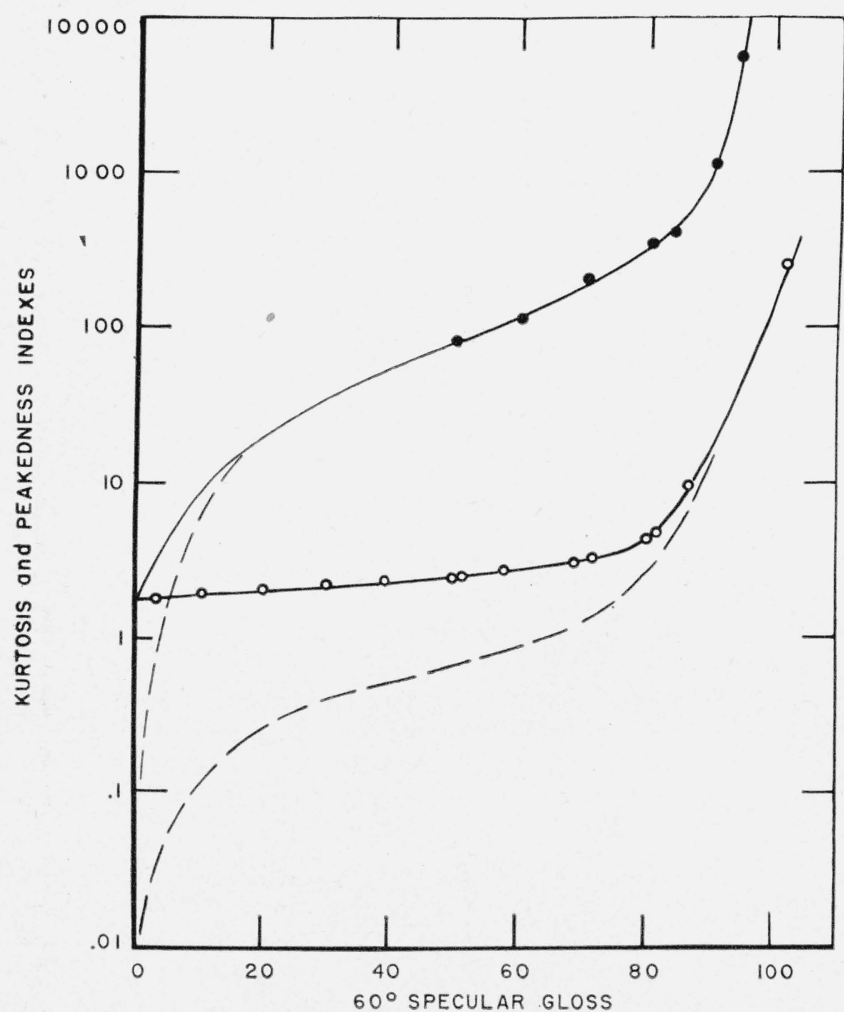

Figure 3. Kurtosis index, $\alpha_{4}$ (solid lines), and peakedness index, $P$ (dashed lines), plotted against $60^{\circ}$ specular gloss.

These indexes differ by a constant amount, 1.78 , which, for the indexes above 10 , is unimportant, so that points for kurtosis index alone are shown above 10 . Note the division of the specimens into two groups: specimens forming sharp images, specimens forming no sharp images, $\bigcirc$. depending on the appearance of the reflected images. Those specimens forming sharp images shaded by varying haze fall into one group. Those specimens forming no sharp images, but having varying specular gloss, fall in the other group. This examination for correlation indicates not only that direct correlation exists, but also that separation into distinct groups in accord with visual appearance is accomplished by the goniophotometric curve analysis, whereas the glossmeter readings alone do not suggest the existence of these two groups.

Correlation of dispersion, $\sigma$, with $60^{\circ}$ specular gloss is shown in figure 4. This figure shows the division of the specimens into the same two groups. The correlation for $\sigma$ is inverse, that is, where $\sigma$ is large, gloss is low, and conversely.

We must conclude from the above data that this analysis not only correlates with existing glossimetry techniques but yields additional information about surface appearance.

\subsection{Selection of Instrumental Geometry}

This analysis has been shown to have advantages over abridged methods in surface-appearance evaluation. Abridged methods of measurement, using

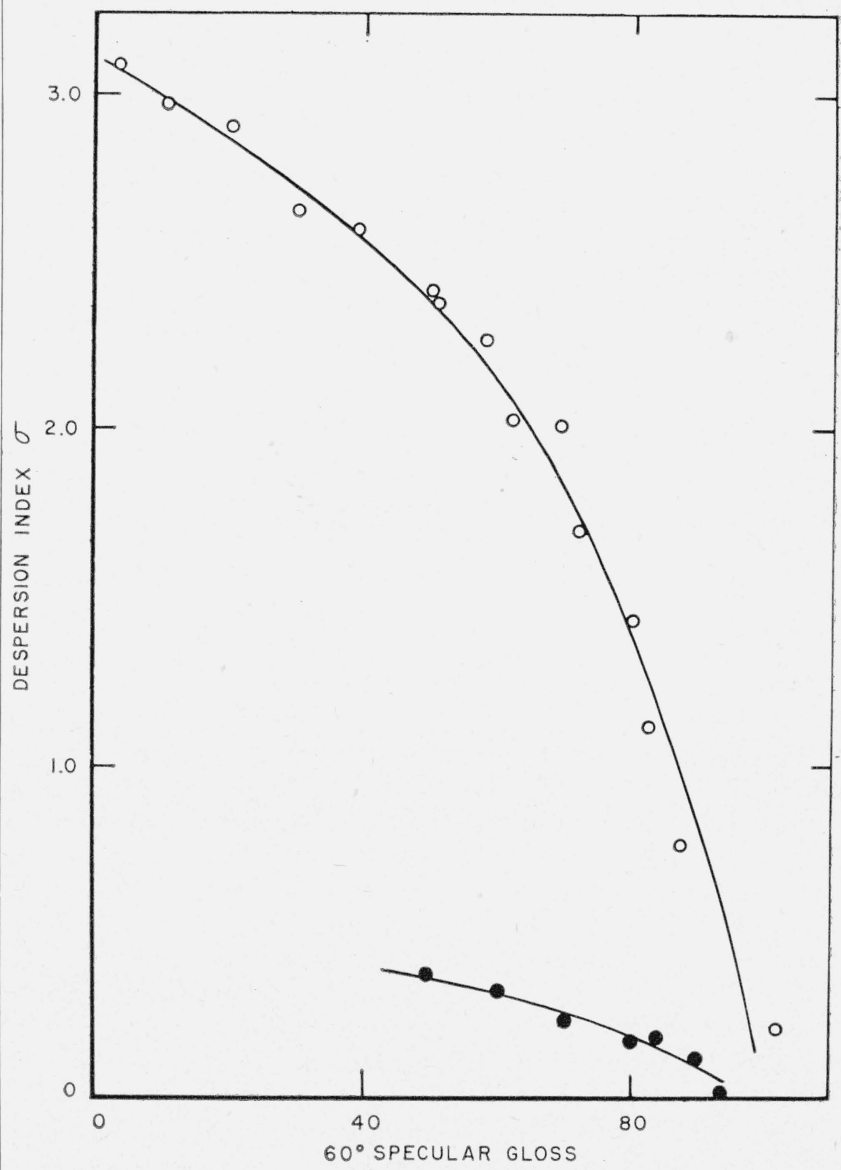

FIGURE 4. Dispersion index, $\sigma$, plotted against $60^{\circ}$ specular gloss.

Specimens forming sharp images, ; specimens forming no sharp images, $\bigcirc$. 
glossmeters, are required to obtain information economically during the production of materials. Glossmeters, as well as goniophotometers, should have geometry to yield optimum discrimination between specimens. Assuming that the receiver is adequately sensitive, the resolution and the incidence angle may be chosen to yield this optimum discrimination.

To determine the optimum geometric conditions for goniophotometry and glossimetry a series of goniophotometric curves should be obtained with various materials for incidence angles ranging from perpendicular to grazing. Then the information from the goniophotometric curves needs to be correlated with visual estimates of gloss. The data for two specimens, $A$ (acid-etched black glass) and $S$ (sand-blasted black glass), having $60^{\circ}$ specular-gloss values of about 80 , will serve to illustrate the procedure for finding optimum geometry (see fig. 5).

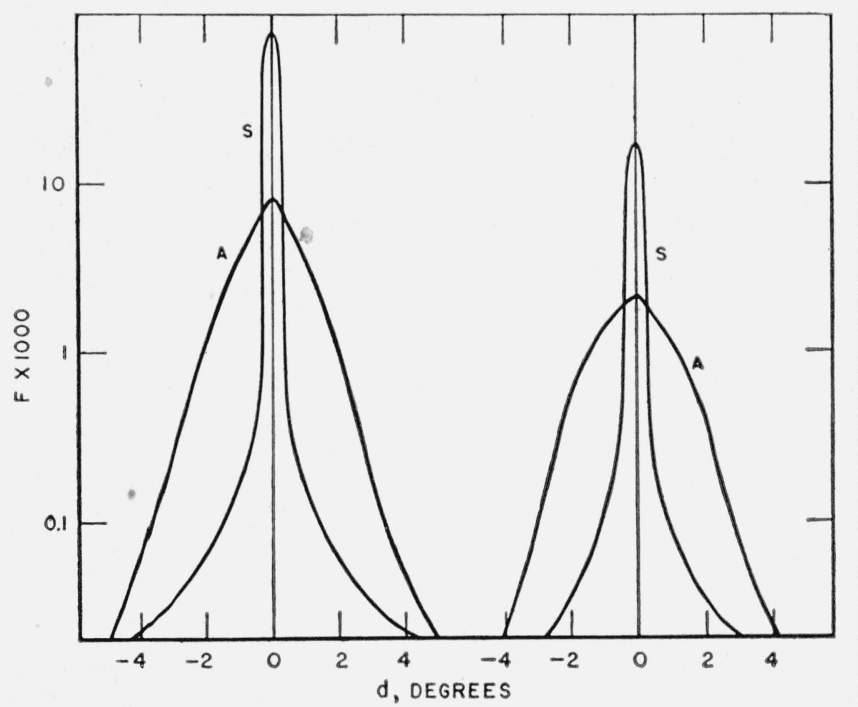

Figure 5. Comparison of $60^{\circ}$ with $20^{\circ}$ incidence angles. $60^{\circ}$ incidence on left; $20^{\circ}$ incidence on right; sand-blasted black glass, $S$; acidetched black glass, $A$

\section{a. Choice of Incidence Angle}

Let us consider first how the peakedness index, $P$, may be used in the problem of choice of incidence angle.

Greatest discrimination between specimens is indicated where the difference between peakedness indexes is greatest. In the illustration, for $20^{\circ}$ incidence, the difference between $P_{S}$ (index for specimen $S$ ) and $P_{A}$ (index for specimen $A$ ) is $P_{S}-P_{A}=153-$ $1.6=151.4$. For $60^{\circ}$ incidence this difference is $P_{S}-P_{A}=379-2.8=376.2$. Thus for greater discrimination between $S$ and $A, 60^{\circ}$ incidence is superior to $20^{\circ}$ incidence for goniophotometric measurements.

This reasoning may be applied to glossmeter measurements. The receiver aperture of a glossmeter includes flux reflected near the specular angle. Thus for optimum discrimination between specimens $S$ and $A$, glossmeter measurements should be made at $60^{\circ}$ rather than at $20^{\circ}$ incidence, provided that the flux accepted for measurement is suffi.. ciently close to the specular angle. To answer the question, what is meant by "sufficiently close", we must consider the problem of choice of receiver aperture size.

\section{b. Choice of Receiver Aperture Size}

Let us now consider how the goniophotometric curves may be used in the problem of choice of receiver aperture size.

The goniophotometric curves provide sufficient information for the choice of receiver aperture size needed for discriminations. For goniophotometric measurements the receiver aperture needs to be sufficiently small to trace adequately the geometric distribution of the fractional reflectances. Gloss.meters, on the other hand, integrate the flux entering the receiver entrance window at the specular angle. The glossmeter must distinguish between different specimens by integrating between the limits of the receiver entrance window the areas under the respective goniophotometric curves. The limits of integration may be evaluated from either of the following relationships:

$$
\begin{array}{r}
\int_{-\alpha}^{a} F_{1} \delta d-\int_{-\alpha}^{a} F_{2} \delta d=K, \\
\int_{-\alpha}^{a} F_{1} \delta d=K \int_{-\alpha}^{a} F_{2} \delta d,
\end{array}
$$

or

where $a$ is the limit of integration, $F_{1}$ and $F_{2}$ are fractional reflectances of two specimens, $d$ is the departure from the specular angle, and $K$ is an experimentally determined constant. Equation (16) states that the difference between the integrated areas is constant. Equation (17) states that the ratio of the integrated areas is constant. The constant $K$ should be evaluated for specimens of similar materials from visual estimates of either gloss differences or gloss ratios. No attempt should be made to evaluate $K$ for radically dissimilar specimen types.

\section{Conclusions}

Since the surface appearance attribute ascribable to gloss may be evaluated most advantageously by monoplane goniophotometric curves, a technique for analyzing these curves needs to be adopted. The variables of classical statistics, dispersion, skewness, and kurtosis, have been studied in this connection, and it is found that the kurtosis index in particular summarizes in a meaningful way the information given by the goniophotometric curve. Correlation between $60^{\circ}$ specular gloss and kurtosis index is fairly good, and indicates that the two measure similar properties of surfaces such as those studied. Furthermore, correlation between distinctness of image and kurtosis index is a decided improvement over correlation between distinctness of image and $60^{\circ}$ specular gloss. Kurtosis index is therefore proposed for adoption as a standard technique for re- 
ducing goniophotometric data to meaningful numbers. This index has been applied successfully for choosing instrumental geometry for abridged goniophotometry, that is, glossimetry.

\section{References}

[1] F. Thaler, Die diffuse Reflexion des Lichtes an matten Oberflachen, Ann. Physik [4] 11, 996 (1903).

[2] P. Bouguer, Optice de diversis luminis gradibus dimentien dis. Opus posthumum in lucem editum a D. de la Caille et in latinum conversum a Joachimo Richtenberg, J. S. Joannis Thomae Trattner. Book 2, section 3 art. $3(1762)$

[3] J. H. Lambert, Photometria sive de mensura gradibus luminis, colorum, et umbrae, Augsburg (1760).

[4] H. J. MeNicholas, Equipment for measuring the reflective and transmissive properties of diffusing media, J. Research NBS 13, 211, (1934) RP704.

[5] L. A. Wetlaufer and W. E. Scott, The measurement of gloss, Ind. Eng. Chem., Anal. Ed. 12, 647 (1940).

[6] H. K. Hammond, III, and I. Nimeroff, Measurement of sixty-degree specular gloss, J. Research NBS 44, 585 (1950) RP2105.

[7] ASTM tentative method of test for $60^{\circ}$ specular gloss of paint finishes, D 523, Book of ASTM Standards, Part 4 (1949).
[8] V. G. W. Harrison, Definition and measurement of gloss, p. 106 (Patra, Cambridge, England, 1945).

[9] W. W. Barkas, Analysis of light scattered from a surface of low gloss into its specular and diffuse components. Proc. Phys. Soc. (London) 51, 274 (1939).

[10] G. I. Pokrowski, Zur Theorie der Diffusen Lichtreflexion, Z. Physik. Part I, 30, p. 66 (1924), Part II, 35, p. 34 (1925), Part III, 35, p. 390 (1926), Part IV, 36, p. 472 (1926).

[11] A. Fresnel, Calcul des teintes que polarization developpe dans lames cristallisees, Ann. chim. phys. 17, 194, 312 (1821).

[12] T. K. Chinmayanandam, On the specular reflection from rough surfaces, Phys. Rev. (London) [II], 13, 96 (1919)

[13] H. L. Reitz, Mathematical Statistics (The Open Court Publishing Co., LaSalle, Ill., 1927).

[14] F. E. Croxton and D. J. Cowden, Applied general statistics (Prentice Hall, New York, N. Y., 1946).

[15] M. P. Pelton, The luster of textile fabrics and a method of measurement, Trans. Opt. Soc. (London) 31, 184 (1930).

[16] R. S. Hunter, Gloss investigations using reflected images of a target pattern, J. Research NBS 16, 357 (1936) RP879.

Washington, February 29, 1952. 